\title{
NMR structure of stem-loop D from human rhinovirus-14
}

\author{
STEPHEN J. HEADEY, ${ }^{1,5}$ HE HUANG,${ }^{2,5,6}$ JOLYON K. CLARIDGE, ${ }^{1}$ GISELLE A. SOARES, ${ }^{1}$ KAUSHIK DUTTA, ${ }^{3}$ \\ MARTIN SCHWALBE, ${ }^{1}$ DAIWEN YANG, ${ }^{4}$ and STEVEN M. PASCAL ${ }^{1}$ \\ ${ }^{1}$ Institute of Fundamental Sciences, Massey University, Palmerston North, New Zealand \\ ${ }^{2}$ Department of Biochemistry and Biophysics, University of Rochester Medical Center, Rochester, New York 14627, USA \\ ${ }^{3}$ New York Structural Biology Center, New York, New York 10027-7556, USA \\ ${ }^{4}$ Department of Biological Sciences, National University of Singapore, Singapore 117543
}

\begin{abstract}
The $5^{\prime}$-cloverleaf of the picornavirus RNA genome is essential for the assembly of a ribonucleoprotein replication complex. Stem-loop D (SLD) of the cloverleaf is the recognition site for the multifunctional viral protein $3 C^{\text {pro }}$. This protein is the principal viral protease, and its interaction with SLD also helps to position the viral RNA-dependent RNA polymerase (3D ${ }^{\text {pol }}$ ) for replication. Human rhinovirus-14 (HRV-14) is distinct from the majority of picornaviruses in that its SLD forms a cUAUg triloop instead of the more common uYACGg tetraloop. This difference appears to be functionally significant, as $3 C^{\text {pro }}$ from tetraloopcontaining viruses cannot bind the HRV-14 SLD. We have determined the solution structure of the HRV-14 SLD using NMR spectroscopy. The structure is predominantly an A-form helix, but with a central pyrimidine-pyrimidine base-paired region and a significantly widened major groove. The stabilizing hydrogen bonding present in the uYACGg tetraloop was not found in the cUAUg triloop. However, the triloop uses different structural elements to present a largely similar surface: sequence and underlying architecture are not conserved, but key aspects of the surface structure are. Important structural differences do exist, though, and may account for the observed cross-isotype binding specificities between $3 C^{\text {pro }}$ and SLD.
\end{abstract}

Keywords: NMR; picornavirus; rhinovirus; RNA; replication; $3 C^{\text {pro }}$

\section{INTRODUCTION}

Human rhinoviruses (HRVs) are members of the picornaviridae family, which also includes poliovirus, hepatitis A virus, foot and mouth virus, and enteroviruses, including the coxsackieviruses. They are also closely related to SARS and other coronaviruses. Rhinoviruses are estimated to be responsible for $>50 \%$ of all respiratory tract infections, and thus are a significant economic pest through missed work (Greenberg 2003). Upper respiratory tract infections can result in complications such as otitis media and sinusitis. HRV infections of the lower respiratory tract are the second most common infectious agents associated with pneumonia and bronchiolitis in young children, and commonly cause exacerbations and hospitalizations in patients with chronic airway diseases, including asthma (Hayden 2004). There are 110 identified HRV serotypes.

\footnotetext{
${ }^{5}$ These authors contributed equally to this work.

${ }^{6}$ Present address: Vaccinex Incorporated, 1895 Mt. Hope Avenue, Rochester, NY, 14620.

Reprint requests to: Steven M. Pascal, Institute of Fundamental Sciences, Massey University, Palmerston North, New Zealand; e-mail: s.pascal@massey.ac.nz; fax: 64-6-3505682.

Article published online ahead of print. Article and publication date are at http://www.rnajournal.org/cgi/doi/10.1261/rna.313707.
}

Like all picornaviruses, HRV-14 possesses a relatively small $(7.2 \mathrm{~kb})$ single-stranded RNA genome that is replicated without the use of a DNA intermediate. The genome is divided into three regions: $5^{\prime}$ and $3^{\prime}$ untranslated regions (UTR), separated by a single gene encoding a $250 \mathrm{kDa}$ polyprotein. After translation, the nascent polyprotein is autolytically cleaved into component proteins that form the viral capsid (VP4, VP2, VP3, and VP1) and those involved in viral replication $\left(2 \mathrm{~A}, 2 \mathrm{~B}, 2 \mathrm{C}, 3 \mathrm{~A}, 3 \mathrm{~B}, 3 \mathrm{C}^{\text {pro }}\right.$, and $\left.3 \mathrm{D}^{\mathrm{pol}}\right)$. The majority of cleavage events are performed by a multifunctional viral protein, $3 \mathrm{C}^{\text {pro }}$, a cysteine protease similar in structure to the serine proteases (Lawrence and Thatch 1975; Pelham 1978; Matthews et al. 1994). In addition to its role in processing viral proteins, $3 \mathrm{C}^{\text {pro }}$ cleaves host proteins involved in mRNA transcription and translation, thereby diverting resources to viral reproduction (Amineva et al. 2004; Kuyumcu-Martinez et al. 2004). $3 \mathrm{C}^{\text {pro }}$ is also intimately involved in viral genome replication (Xiang et al. 1995). 3C $\mathrm{C}^{\text {pro }}$ for a time remains covalently joined to the RNA-dependent RNA polymerase (3Dpol) to form the 3CD fusion protein. 3CD binds to RNA stem-loop D (SLD), near the start of the 5'-UTR, through a mechanism that is not completely understood, but is thought to involve positively charged residues of $3 \mathrm{C}^{\text {pro }}$ located on the surface opposite to its protease active site 
(Andino et al. 1990; Leong et al. 1993; Matthews et al. 1994; Walker et al. 1995). The interaction of 3CD with SLD is essential for the assembly of the ribonucleoprotein complex and the initiation of transcription (Xiang et al. 1995). Host proteins are recruited to the process, including the $\operatorname{poly}(\mathrm{A})$ binding protein (PABP), which can bind both to the poly(A) tail of the short 3' UTR and to the $5^{\prime}$ ribonucleoprotein complex. This suggests that the viral RNA may be effectively circularized prior to replication (Meredith et al. 1999; Herold and Andino 2001).

While viral genomes with a deleted 3' UTR still produce viable viruses, albeit with greatly reduced efficiency (Sarnow 1989; Todd and Semler 1996), mutations in $3 C^{\text {pro }}$ or SLD that abrogate their interaction abolish viral reproduction (Walker et al. 1995; Zell et al. 2002). HRV-14 is distinct from most picornaviridae and the majority of HRV isotypes in containing a triloop (Fig. 1A) instead of the more common tetraloop (Fig. $1 B, C)$ in its SLD. Intriguingly, although recombinant HRV$143 \mathrm{C}^{\text {pro }}$ can bind the tetraloop SLD of poliovirus (Walker et al. 1995; Blair et al. 1998), recombinant $3 C^{\text {pro }}$ from poliovirus or coxsackievirus B3 (CVB3) cannot bind to HRV-14 SLD (Xiang et al. 1995; Blair et al. 1998; Zell et al. 2002; Rieder et al. 2003). Substituting the HRV-14 SLD triloop with certain tetraloop sequences results in a stemloop that can interact with CVB3 $3 C^{\text {pro }}$ (Rieder et al. 2003). Taken together, these observations suggest that important differences between tetraloop and triloop structures can affect $3 \mathrm{C}^{\text {pro }}$ binding, but also suggest that important similarities must exist as well.

We have used NMR spectroscopy to determine the solution structure of the 27 nucleotide (nt) HRV-14 SLD region sufficient for $3 \mathrm{C}^{\text {pro }}$ binding (Walker et al. 1995). Comparison with recently studied tetraloop SLDs (Du et al. 2003, 2004; Ohlenschlager et al. 2004) shows that the HRV-14 triloop SLD does indeed retain many of the structural features of the tetraloop SLDs while also possessing distinct characteristics that may account for its unique $3 \mathrm{C}^{\text {pro }}$ binding properties.

\section{RESULTS}

\section{Exchangeable proton resonance assignment and interpretation}

The HRV-14 SLD 27mer contains 16 imino protons, of which 13 were observed in ${ }^{1} \mathrm{H} 1 \mathrm{D}$ and ${ }^{1} \mathrm{H},{ }^{1} \mathrm{H}$ 2D NOESY spectra in $\mathrm{H}_{2} \mathrm{O}$ (Fig. 2), consistent with their participation in hydrogen-bonded base pairs. Nine of these 13 resonances lie in the spectral range typically found in standard Watson-Crick base pairing (12.5-14 ppm) and were assigned to stem I and stem II nucleotides. The four imino resonances lying outside of this range, two each at $\sim 10.0$ and $11.4 \mathrm{ppm}$, display unusually strong imino-imino NOE cross-peaks. Weak NOE connectivities to bases in the Watson-Crick regions enabled unambiguous assignment of these resonances to uracil nucleotide participating U-U mismatched base pairs in the central region of the helix (Fig. 2). The imino resonance of G1 was not observed, suggesting that the G1-C27 base pair does not form or is not sufficiently shielded from solvent to prevent fast imino proton exchange on the NMR time scale. Similarly, no imino resonance could be assigned for $\mathrm{U} 22$, which might be expected to participate in a noncanonical CU base pair in the central region of the helix. However, this observation is consistent with studies of other SLDs where the homologous imino resonance is either not observed (Du et al. 2003; Ohlenschlager et al. 2004) or only detected at low temperature ( $\mathrm{Du}$ et al. 2004). The absence of detectable imino resonances for U13 and U15 is consistent with the location of these bases in a loop region where they do not base pair.

\section{Nonexchangeable resonance assignments}

Assignment of stem II and triloop resonances of the $27 \mathrm{mer}$ was greatly assisted by reference to previously published assignments of a $14 \mathrm{nt}$ region of HRV-14 SLD (Huang et al. 


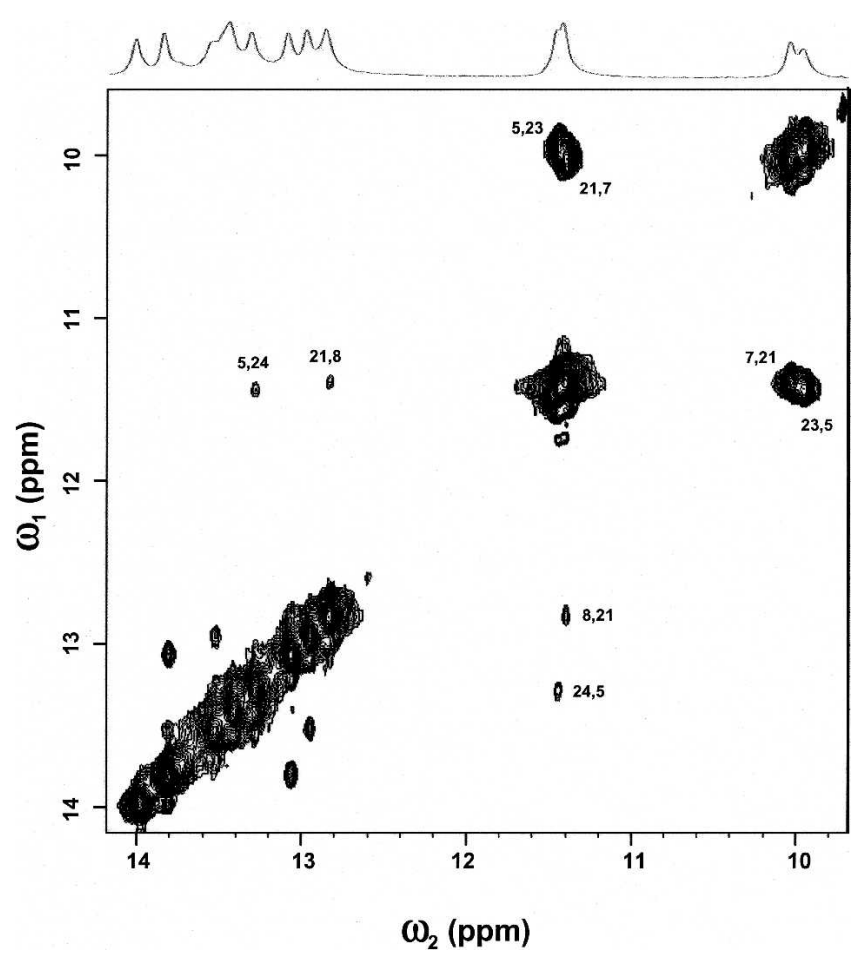

FIGURE 2. Portion of $700 \mathrm{MHz}$ 2D NOESY spectrum of HRV-14 SLD in $\mathrm{H}_{2} \mathrm{O}$ containing imino-imino connectivities. A $1 \mathrm{D}{ }^{1} \mathrm{H}$ NMR spectrum of the full imino region is plotted across the top. Key crosspeaks are labeled by nucleotide number.

2001), which included nucleotides $8-20$ of Figure 1 A. The chemical shifts of nucleotides 9-19 showed little departure from the previous assignments. All nonexchangeable base resonances as well as all sugar $\mathrm{H}^{\prime}$ and $\mathrm{H} 2^{\prime}$ resonances, with the exception of the $\mathrm{H}^{\prime}$ of $\mathrm{G} 1$, were assigned. Of the remaining sugar resonances 23 of the $27 \mathrm{H3}^{\prime}, 24$ of the $27 \mathrm{H}^{\prime}$ and 18 of the $54 \mathrm{H}^{\prime}$ and $\mathrm{H}^{\prime \prime}$ resonances were assigned. In total, 167 of the 211 nonexchangeable ${ }^{1} \mathrm{H}$ resonances of the $27 \mathrm{mer}$ were unambiguously assigned. The extent of assignment was higher in the pyrimidine-mismatch $(83 \%)$ and triloop (92\%) regions than in stem I (71\%) or stem II (79\%). Strong intranucleotide and sequential $\left(\mathrm{H}^{\prime}{ }^{\prime}\right)-\left(\mathrm{H} 6\right.$ or H8) and $\left(\mathrm{H}^{\prime}{ }^{\prime}\right)-(\mathrm{H} 6$ or H8) NOE crosspeaks observed for most nucleotides are consistent with the classical base stacking of RNA double helices.

\section{Sugar conformations and backbone geometry}

DQF-COSY spectra show strong intranucleotide $\left(\mathrm{H}^{\prime}{ }^{\prime}\right)-$ $\left(\mathrm{H} 2^{\prime}\right)$ multiplets for U13, A14, and U15 of the triloop, consistent with predominantly $\mathrm{C}^{\prime}$ '-endo sugar pucker. The ribose rings of these nucleotides were therefore constrained to $\mathrm{C} 2^{\prime}$-endo during structure calculations. The nucleotides of the closing base pair of the triloop (C12-G16), as well as $\mathrm{C} 27$, have weak but detectable $\left(\mathrm{H} 1^{\prime}\right)-\left(\mathrm{H} 2^{\prime}\right)$ multiplets, typical of a mixture of $\mathrm{C}^{\prime}$-endo and $\mathrm{C}^{\prime}$-endo sugar puckers. Therefore, the sugar conformations of these nucleotides were not constrained. The absence of $\left(\mathrm{H} 1^{\prime}\right)-$ $\left(\mathrm{H} 2^{\prime}\right)$ multiplets for the sugars of other nucleotides, including those of the mismatch region, is consistent with the C3'-endo conformation found in A-form RNA. C3'endo constraints were consequently applied to these residues during structure calculations. The intensities of intranucleotide NOEs between $\left(\mathrm{H}^{\prime}\right)$ and aromatic protons provided no evidence of syn glycosidic bond angles for any nucleotide. The ${ }^{1} \mathrm{H},{ }^{31} \mathrm{P}$-HETCOR revealed significantly downfield shifted ${ }^{31} \mathrm{P}$ resonances for $\mathrm{U} 15, \mathrm{U} 17$, and to a lesser extent, U13 and G16, indicating possible departures from A-form backbone geometry in these regions.

\section{Structural calculation}

The solution structure of HRV-14 SLD is shown in Figure 3A. Structural statistics are given in Table 1. HRV-14 SLD is comprised of two A-form helical stem regions containing standard Watson-Crick base pairs. These stems are separated by a pyrimidine-mismatch region containing a $\mathrm{C}-\mathrm{U}$ base pair flanked by two $\mathrm{U}-\mathrm{U}$ base pairs. The structure is capped by a hairpin triloop. The ribose moieties of U13, A14, and U15 of the triloop and G16 are in the
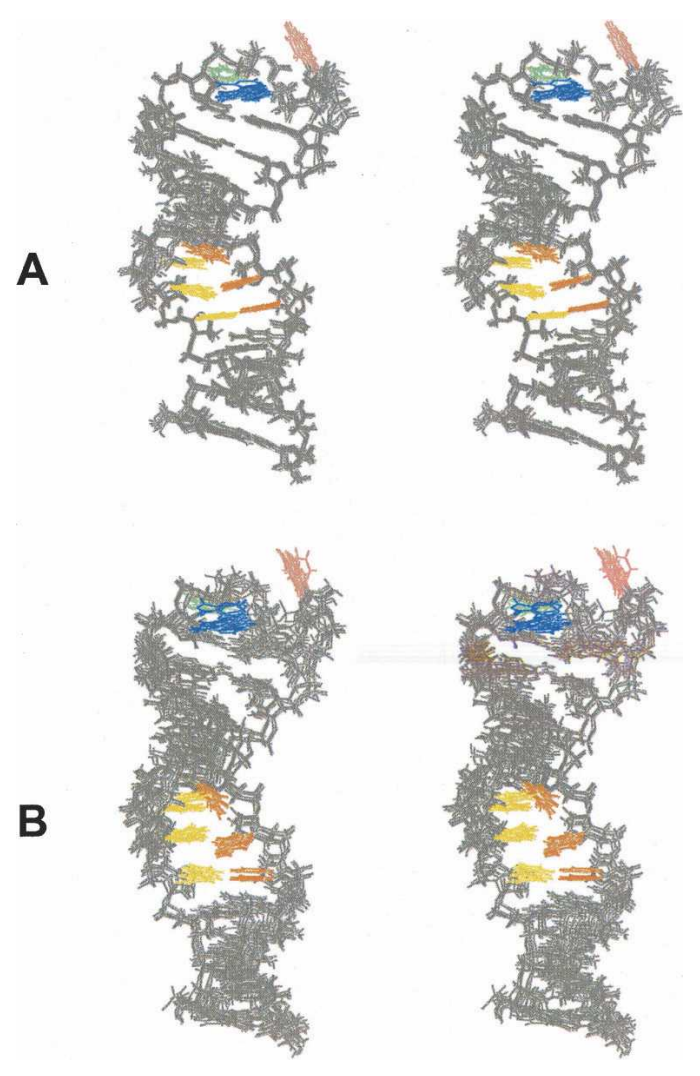

FIGURE 3. Stereo views of the superposition of the 10 lowest-energy structures of HRV-14 SLD determined $(A)$ with RDCs, and $(B)$ without RDCs. Bases are colored as in Figure 1A. 
TABLE 1. Structural and refinement statistics of HRV-14 SLD

\begin{tabular}{lc}
\hline Statistics & \\
\hline Total number of constraints & $655(\sim 24 / \mathrm{nt})$ \\
NOE constraints & 358 \\
Intranucleotide NOE constraints & 202 \\
Internucleotide NOE constraints & 156 \\
Backbone torsion angle constraints & 85 \\
Sugar pucker constraints & 96 \\
Hydrogen bond constraints & 40 \\
Residual dipolar couplings & 66 \\
Base planarity & 10 \\
Number of violations & \\
NOE viol. $>0.2 \AA$ & 0 \\
torsion angle viol. $>5^{\circ}$ & 0 \\
dipolar coupling viol. $>2.0 \mathrm{~Hz}$ & \\
Mean deviation from ideal covalent geometry & $0.0017 \pm 0.00004$ \\
Bonds $(\AA)$ & $0.6565 \pm 0.0082$ \\
Angles $\left(^{\circ}\right)$ & $0.6017 \pm 0.0235$ \\
Impropers $\left({ }^{\circ}\right)$ & \\
Pairwise heavy atom RMSD ( $\AA$ ) & $0.59 \pm 0.16$ \\
All $(1-27)$ & $0.26 \pm 0.08$ \\
Stem I (1-4, 24-27) & $0.38 \pm 0.19$ \\
Mismatch $(5-7,21-23)$ & $0.51 \pm 0.18$ \\
Stem II $(8-12,16-20)$ & $0.52 \pm 0.31$ \\
Triloop $(13-15)$ & \\
\hline
\end{tabular}

$\mathrm{C} 2$ '-endo conformation, whereas those of the stem and mismatch regions and $\mathrm{C} 12$ are in the $\mathrm{C} 3{ }^{\prime}$-endo conformation typical of A-form RNA. All glycosidic bonds are in the more common trans conformation. The solution structure is relatively well defined (pair-wise heavy atom RMSD = $0.59 \pm 0.16 \AA$ ).

\section{Pyrimidine-mismatch conformation}

The central mismatch region of SLD is pyrimidine-rich, containing the sequences UCU and UUU (Fig. 1A). Hydrogen bonding between $\mathrm{U}-\mathrm{U}$ base pairs is evident from the presence of unusual imino-imino NOEs as discussed above and shown in Figure 2. This pattern arises from the imino proton of U5/U21 ( 10.0 ppm) hydrogen bonding to the $\mathrm{O} 4$ of $\mathrm{U} 23 / \mathrm{U} 7$, respectively, while the imino of $\mathrm{U} 23 /$ $\mathrm{U} 7$ ( $\sim 11.4 \mathrm{ppm})$ hydrogen bonds to the $\mathrm{O} 2$ of U5/U21, respectively (see Fig. 4). The intervening C6-U22 base pair has hydrogen bonds between the amino group of C6 and O4 of U22 and also between the imino proton of U22 and N6 of C6. No hydrogen bond constraints were included in structure calculations for this base pair, as its labile protons were not observed in NOE spectra. The incorporation of RDC constraints appears to have been critical in maintaining correct hydrogen bond geometry for C6-U22, and in structures calculated without RDCs this base pair is not well formed (Fig. 3B). RDC constraints also encourage base planarity for the U7-U21 pair. Although A-form geometry is largely maintained in the pyrimidine-mismatch region, the minor groove width is reduced by $\sim 2-3 \AA$ relative to stems I and II. This reduction is sufficient to explain the observed $\mathrm{C} 6\left(\mathrm{H} 1^{\prime}\right)-\mathrm{U} 23\left(\mathrm{H} 1^{\prime}\right)$ NOE.

\section{Triloop conformation}

The triloop (Fig. 5A) is comprised of $\mathrm{cU}_{13} \mathrm{~A}_{14} \mathrm{U}_{15}$ g. The $\mathrm{U} 13$ base is in the major groove where it stacks onto the $\mathrm{C} 12$ base, giving rise to relatively strong sequential NOEs from both $\mathrm{C} 12\left(\mathrm{H}^{\prime}\right)$ and $\mathrm{C} 12\left(\mathrm{H}_{2}^{\prime}\right)$ to $\mathrm{U} 13(\mathrm{H} 6)$ that are comparable in intensity to analogous NOEs in the stem regions (Fig. 5B). However, a less typical NOE of moderate intensity between $\mathrm{C} 12\left(\mathrm{H}^{\prime}\right)$ and $\mathrm{U} 13(\mathrm{H} 5)$ supports an enhanced degree of base stacking. In contrast, the sequential $\mathrm{H} 1^{\prime}$-aromatic NOEs of U13 to A14, A14 to U15, and $\mathrm{U} 15$ to G16 are very weak, which is consistent with the lack of base stacking for these pairs of nucleotides. The base of A14 is located in the minor groove. Its position is constrained by a network of NOEs to A14 base protons, including $\mathrm{A} 14(\mathrm{H} 2)$ and $\mathrm{A} 14(\mathrm{H} 8)$ resonances to $\mathrm{U} 13\left(\mathrm{H} 4^{\prime}\right)$. The $\mathrm{U} 15$ base is somewhat disordered, and has relatively few internucleotide NOE connectivities. The incorporation of three RDC constraints for this nucleotide stabilized its base in a position tilted toward the backbone in the direction of A14. The closing base pair (C12, G16) forms a nearly standard Watson-Crick pair but with the sugar of G16 adopting a C2' -endo conformation. As mentioned, both C12 and G16 show weak $\left(\mathrm{H}^{\prime}{ }^{\prime}\right)-\left(\mathrm{H} 2^{\prime}\right)$ multiplets in COSY spectra, which suggest mixed sugar puckers, and consequently, no sugar pucker constraints were applied to these nucleotides.

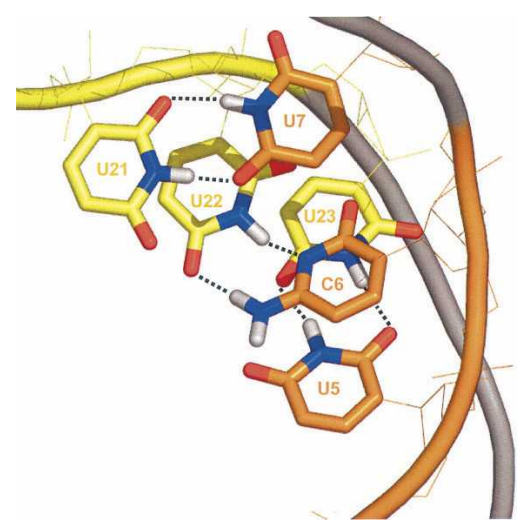

FIGURE 4. A zoom view of the pyrimidine-pyrimidine-mismatch region. Base-pairing hydrogen bonds are indicated by dotted lines, while base hydrogens that do not participate in hydrogen bonds are omitted. Each of the two U-U base pairs form an H3-O2 and an O4$\mathrm{H} 3$ hydrogen bond. However, in the lower pairing (U5-U23), the $\mathrm{O} 2$ is from U5 while the $\mathrm{O} 4$ is from $\mathrm{U} 23$. In the top pairing, the $\mathrm{O} 2$ is from U21 while the $\mathrm{O} 4$ is from U7. The central C6-U22 base pair forms hydrogen bonds from C6(amino group) to $\mathrm{U} 22(\mathrm{O} 4)$ and from $\mathrm{U} 22(\mathrm{H} 3)$ to $\mathrm{C} 6(\mathrm{~N} 3)$. The phoshpodiester backbone is shown as a worm, and the color scheme is that of Figure 1A, with the addition of red, white, and blue for base oxygen, hydrogen, and nitrogen atoms, respectively. 


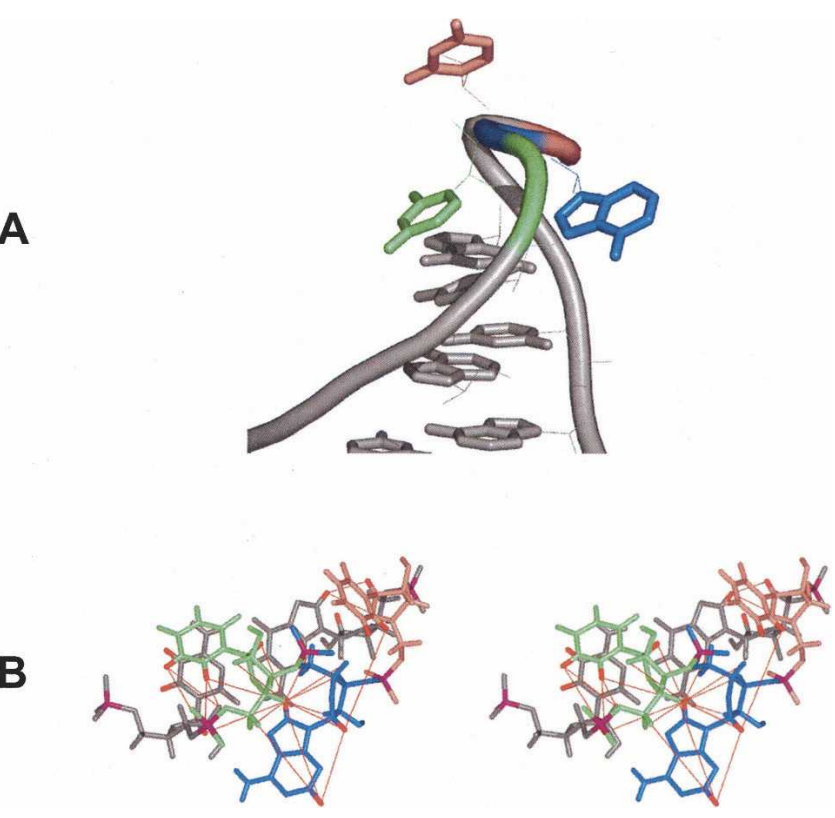

FIGURE 5. The HRV-14 SLD triloop. (A) Cartoon representation. U13 (green) lies on the major groove side, while A14 (blue) lies along the minor groove. U15 is shown in pink. (B) Stereo view showing key NOE connectivities (red lines) and $\mathrm{CH}$ bonds specifically oriented by RDC data (red bonds). Nucleotides are otherwise colored as in Figure $1 \mathrm{~A}$, with the addition of violet phosphorous atoms to assist in viewing the path of the backbone.

However, in each of the 10 lowest-energy structures, the G16 and C12 sugars adopt C2'-endo and C3' -endo conformations, respectively.

\section{DISCUSSION}

\section{Advantage of RDC analysis}

Not surprisingly, the precision of the structural family determined with RDCs (Fig. 3A) is significantly higher than that of the family determined without RDCs (Fig. 3B), with pair-wise RMSDs of 0.59 and $1.00 \AA$, respectively. Due to the planarity of nucleic acid bases, the presence of base pairing, and the restricted conformational space available to the cyclic ribose moiety, a modest number of RDCs is sufficient to afford a large increase in precision (Mauffret et al. 2002). Moreover, the RDC-refined structures presented here are more compact, with a mean axial length of $47 \AA$ compared to $52 \AA$ for the non-RDC refined structures. Similar shortening of the helix upon refinement with RDCs also occurred in structure calculations for the consensus SLD (Du et al. 2004), suggesting that at least in some cases, elongated structures may be an inherent bias of structure calculation algorithms performed without RDCs.

A portion of the SLD structure presented here is a refinement of a previously reported stem-loop structure (Huang et al. 2001), which included stem II and the triloop, but not stem I or the mismatch region. In this previous study (of a 14mer), performed without the benefit of RDC data, the A14 base was thought to occupy the major groove. Current structural analysis (of a 27mer) places the A14 base in the minor groove. When RDC restraints are omitted from the present refinement, the base of A14 resides in the major groove in $30 \%$ of structures, but in the minor groove in the remaining $70 \%$ of acceptable structures. This second possibility, that the A14 base may occupy the minor groove, was only identified upon inclusion of additional NOEs observed with the $27 \mathrm{mer}$, including from the previously unassigned $\mathrm{A} 14(\mathrm{H} 2)$ to the $\mathrm{H} 4^{\prime}$ and $\mathrm{H}^{\prime \prime}$ resonances of U13. These two potential conformational groups were then used to back-calculate NOEs. The results favored the A14 base residing in the minor groove. Importantly, the incorporation of RDC data conclusively precluded the major groove configuration without necessitating recourse to NOE back-calculation. These findings underscore the utility of RDC data for resolving structural ambiguities in nucleic acids, particularly in nonstandard loop regions.

\section{HRV-14 SLD is an atypical A helix}

Although HRV-14 SLD is unequivocally an A-form helix, its geometry nevertheless shows significant divergence from idealized A form. The defining features of an A helix, namely, the $3^{\prime}$-endo sugar pucker, off-axis positioning of the bases, and the inclination of the bases relative to the helical axis, are all present in HRV-14 SLD. A local cinching of the minor groove occurs in the pyrimidine-mismatch region, presumably as a result of the smaller base sizes. The minor groove width in this region decreases from the typical A-form distance of $11 \AA$ to $\sim 8 \AA$. Beginning at the pyrimidine-mismatch region the major groove width progressively expands from $7 \AA$, which already exceeds the nominal value for the A-form helix, to $\sim 13 \AA$ (Figs. 6A, $7 \mathrm{~A}$ ), which is in the typical range of a $\mathrm{B}$ helix commonly found in DNA. Such widening of the major groove is a recurrent feature of protein recognition sites, as the major groove of the idealized A helix is too narrow to allow the interrogation of the bases by protein alpha helices. Somewhat similar helical geometry is also evident in the NMR structures of tetraloop SLDs (Figs. 6B,C). In each case, part of the major groove is widened. In the case of the consensus SLD (Du et al. 2004), the presence of two bulged bases in stem I (see Fig. 1B) coincides with the initial expansion of the major groove, which widens to $\sim 12 \AA$ as the cinch is approached, and maintains close to the B-form width thereafter. The coxsackievirus B3 SLD major groove (Ohlenschlager et al. 2004) approaches the B-form width in both stem regions but narrows to an intermediate value in the mismatch region. It is not immediately obvious to what extent groove width differences between these three structures are due to (1) the presence of a bulge in the 

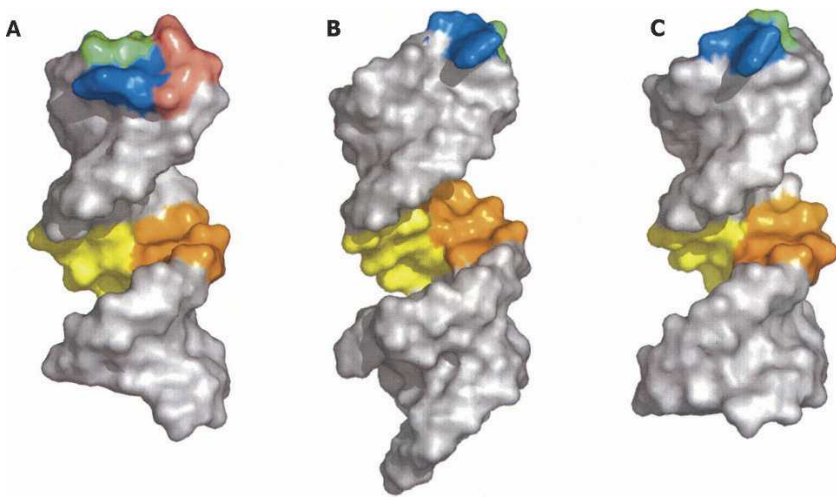

FIGURE 6. Space-filling views of NMR-determined structures of SLD from (A) HRV-14, (B) consensus sequence (Du et al. 2004), and (C) Coxsackievirus B3 (CVB3) (Ohlenschlager et al. 2004). Widened major grooves are evident at the center of each part of the figure. Sequences and colors are as in Figure 1A-C, respectively.

consensus sequence as discussed above; (2) seemingly minor sequence variations within the stems; or (3) differences in technique, such as the inclusion of RDC data in the HRV-14 and consensus structures, but not the coxsackie structure. It is worth noting that Ohlenschlager et al. (2004) found chemical shift perturbations induced by $3 \mathrm{CD}$ binding in the distal end of the mismatch region, stem II, and the apical loop of coxsackievirus B3 SLD. Enhanced access to the major groove in this region is evident in all three SLD structures. Moreover, the U-rich pyrimidine-mismatch region would present an area of high electronegativity that could interact with the basic residues of the putative RNA binding site on $3 C^{\text {pro }}$.

\section{Triloops and tetraloops}

The mechanism of picornaviral replication is so well conserved that picornaviruses with chimeric genomes, where the cloverleafs have been swapped between picornavirus isoptypes, can produce viable virus particles (Rohll et al. 1994; Xiang et al. 1995; Johnson and Semler 1988). It is not surprising, therefore, that recombinant $3 \mathrm{C}^{\text {pro }}$ from picornaviruses with tetraloop SLDs can bind each others' SLDs with near-normal affinity (Zell et al. 2002). However, attempts to identify key binding determinants via mutagenesis have not been completely successful, leading to the suggestion that conserved structural features, rather than conserved nucleotide sequences, are critical for the interaction (Zell et al. 2002; Rieder et al. 2003).

An insight into these conserved features comes from an examination of the tetraloop structures of the consensus (Du et al. 2004) and CVB3 (Du et al. 2003; Ohlenschlager et al. 2004) SLDs. These two stem-loops contain nearly identical tetraloop structures, with base stacking and hydrogen bonding very similar to that previously seen in cUUCGg tetraloops (e.g., Allain and Varani 1995). Tetraloops are common motifs for RNA-RNA and RNA-protein interactions. The importance of the SLD tetraloop in $3 \mathrm{C}^{\text {pro }}$ binding is reinforced by NMR chemical shift perturbation results as discussed above (Ohlenschlager et al. 2004).

HRV-14 is atypical of picornaviruses in that its SLD contains a triloop instead of the more common tetraloop. This difference has proven to be both useful and puzzling in investigations of $3 \mathrm{C}^{\text {pro }}-\mathrm{SLD}$ interactions. The HRV-14 SLD has to this point not been shown capable of interacting with a $3 C^{\text {pro }}$ from a virus whose SLD forms a tetraloop (Xiang et al. 1995; Blair et al. 1998; Zell et al. 2002; Rieder et al. 2003), nor can the corresponding chimeric picornavirus produce viable particles. Interestingly, the reverse is not true, as HRV-14 $3 \mathrm{C}^{\text {pro }}$ can form a stable complex with the tetraloop SLDs from poliovirus (Walker et al. 1995). These observations strongly suggest that the loop region forms part of the $3 \mathrm{C}^{\text {pro }}$ binding site. On the other hand, the lack of binding reciprocity has been difficult to explain.

When the NMR structure of the HRV-14 $c\left(\mathrm{U}_{13} \mathrm{~A}_{14} \mathrm{U}_{15}\right) \mathrm{g}$ triloop (Fig. 8A) is compared to that of the
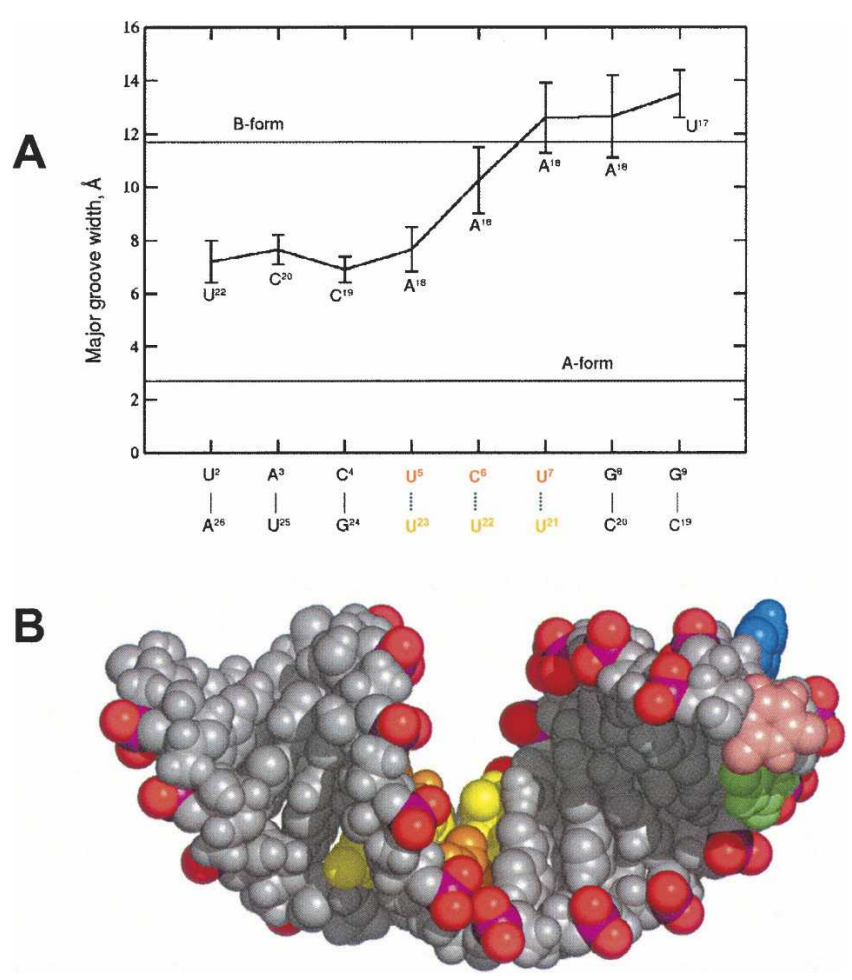

FIGURE 7. (A) Plot of HRV-14 SLD major groove width, calculated as distance of closest phosphorous-phosphorous approach across the groove, minus 5.6 $\AA$. Distances have been measured from the nucleotides listed directly below the $\mathrm{x}$ axis to the nucleotides listed below the error bars. Groove widths for the standard A and B forms are shown as horizontal lines within the figure. Base-paired nucleotides and coloring of the mismatch region have been added below the $\mathrm{x}$ axis for clarity. (B) A CPK rendering of HRV-14 SLD. Bases are again colored as in Figure 1A. Backbone phosphorous nuclei are shown in purple, while backbone oxygens not covalently bound to sugar carbons are shown in red. The widening of the major groove upon approach to the triloop is clearly evident. 

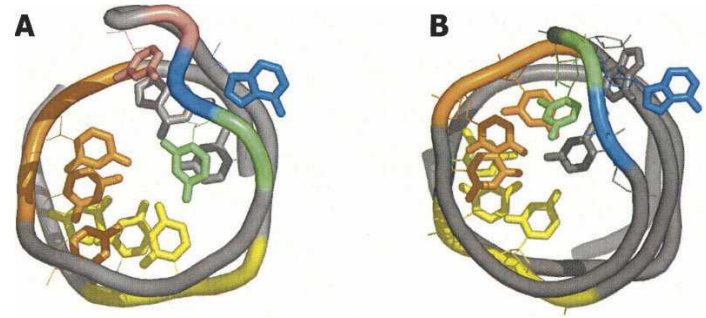

FIGURE 8. (A) Top view of HRV-14 SLD showing sugars and bases only of the nucleotides from the triloop, the central mismatch (orange and yellow), and the closing base pair (C12-G16). In this view, the triloop is closest to the observer. U13 (green), which lies in the major groove side, stacks onto C12 (gray). A14 (blue) lies on the minor groove side. (B) Similar view of the consensus SLD structure (see Fig. 1B). The closing base pair (U12-G16) is omitted for clarity. NMR structure of HRV-14 stem-loop D. However, the tetraloop base pair formed by C13-G15.2 (gray) plays a somewhat analogous role. A14 (blue) again lies along the minor groove, while C15.1 (green) lies on the major groove side. There is no base analogous to U15 from HRV-14 (pink base in $A$ ). (C) The CVB3 SLD structure (see Fig. 1C) shows similarity to the consensus structure in $B$.

$\mathrm{u}\left(\mathrm{Y}_{13} \mathrm{~A}_{14} \mathrm{C}_{15.1} \mathrm{G}_{15.2}\right) \mathrm{g}$ tetraloop (Figs. 8B,C), a striking similarity is apparent. In each case, the A14 base (blue) lies on the minor groove side while a pyrimidine (green) lies in the major groove where it stacks upon paired bases (gray). These shared features could help account for the ability of HRV-14 $3 C^{\text {pro }}$ to bind the tetraloop poliovirus SLD (Walker et al. 1995). However, upon closer examination, this mimicry is achieved via dissimilar underlying architecture. The tetraloops form a noncanonical (Y13G15.2) base pair (gray), with G15.2 in syn conformation and forming a hydrogen bond to the Y13 2'-hydroxyl (see Fig. 1B,C for numbering). Only two unpaired nucleotides are present: A14 (blue) and C15.1 (green). The latter of these is the pyrimidine lying in the major groove, where it stacks onto Y13. The HRV-14 triloop contains no such base pairing: instead of two unpaired bases, there are three. The terminal base pair of stem II (C12, G16) assumes a role similar to the tetraloop (Y13, G15.2) pair. The pyrimidine in the major groove is U13 rather than C15.1, and it stacks onto the $\mathrm{C} 12$ base instead of Y13. In effect, the triloop stem II is one base pair shorter.

The third unpaired base in the triloop is U15. In constrast to U13 and A14, the position of this base has no homolog in the tetraloop structure. Comparatively few internucleotide NOE distance constraints were assigned for this nucleotide, and thus it is somewhat disordered. With the inclusion of RDCs its base is constrained to a position not approaching either groove, but tilted toward the backbone of A14 (Figs. 5A,B). No hydrogen-bonding interactions appear to stabilize this position. It is thus conceivable that $\mathrm{U} 15$ and perhaps other nucleotides of the triloop are, in fact, more disordered than the RDC-refined structures suggest, presumably on a rapid time scale, and that structures calculated using conformationally averaged RDCs and NOEs could provide a false impression of a welldefined conformation. The presence of partially resolved $\mathrm{H} 1^{\prime}-\mathrm{H} 2^{\prime}$ multiplets for the closing base-pair C12-G16 in

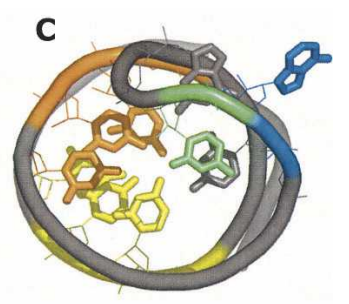

the DQFCOSY spectrum lends some support to this interpretation. RDCs are sensitive to motions on a wide range of time scales, and should tend toward zero if the motions are sufficiently unrestricted. The mean magnitude of the RDCs in the triloop $(5.9 \pm 2.26 \mathrm{~Hz})$ is indeed less than the mean for the rest of the molecule $(10.5 \pm 7.12 \mathrm{~Hz})$. However, these differences may simply reflect the fact that these nucleotides are in a loop region where their orientations would be expected to differ from nucleotides in helical regions. No nucleotide has strongly attenuated RDCs, suggesting that the triloop bases do have preferred orientations. The NOE data are also well fit with single conformers: conformationally averaged models are not required to account for conflicting NOE-derived distances. Taken together, the data would seem to be inconsistent with large structural fluctuations that might lead to, for instance, interconversion of the loop bases between grooves. Nonetheless, until dynamics data are available, judgment should be reserved as to whether the relatively well-ordered RDCrefined structures (Fig. 3A), or the less-ordered structures generated without RDCs (Fig. 3B), provide a truer representation of the conformational space sampled by the triloop.

Regardless of the presence or absence of disorder at this base, U15 clearly does not fit into the structural pattern established by the tetraloops. This fact, together with the ability of HRV-14 $3 \mathrm{C}^{\text {pro }}$ to bind tetraloop SLDs, could suggest that $\mathrm{U} 15$ is not a critical binding determinant in HRV-14. However, this additional base may account for the inability of $3 \mathrm{C}$ proteases from other picornaviruses to bind the HRV-14 SLD (Xiang et al. 1995; Blair et al. 1998; Zell et al. 2002; Rieder et al. 2003) by providing steric or other forms of interference. This contention is supported by point mutagenesis of a replication-incompetent HRV14/poliovirus chimeric genome containing an HRV-14 SLD triloop sequence (Rieder et al. 2003). Creation of a tetraloop via insertion of a guanidine (cUAU $\underline{G}$ ) fails to rescue replication. However, the further $\mathrm{U} \rightarrow \mathrm{C}$ mutation (cUACGg) yields a replicating genome. In the tetraloop SLD structures, the position of this cytosine in the major groove is stabilized by a hydrogen bond from its amino group to the pro $(\mathrm{R})$ phosphate oxygen of the preceding adenosine. Uracil lacks this amino group. Hence, it may be free to leave the major groove, perhaps adopting a conformation similar to U15 in the HRV-14 SLD, and thus interfering with $3 \mathrm{C}^{\text {pro }}$ binding.

In summary, comparisons between the NMR structure of the HRV-14 triloop and those of tetraloop SLDs have been 
made with a view to discerning the conserved features required for $3 \mathrm{C}^{\text {pro }}$ binding. The likely candidates are three structural features: (a) a widened major groove; (b) a basestacked pyrimidine in the major groove; and (c) an adenosine in the minor groove.

\section{MATERIALS AND METHODS}

\section{RNA preparation}

Natural abundance 5'-GUACUCUGGUACUAUGUACCUUU GUAC-3' corresponding to stem-loop D of HRV-14 was purchased from Dharmacon after chemical synthesis, gel electrophoresis, and HPLC purification. The $3^{\prime}$-O-ester protection was removed with $100 \mathrm{mM}$ TEMED-acetate (tetramethylethylenediamine), $\mathrm{pH} 3.8$, as described by the manufacturer. The oligomer was further purified with ethanol precipitation and gel filtration, and buffer exchanged to $90 \mathrm{mM} \mathrm{NaCl}, 10 \mathrm{mM}$ sodium phosphate, $0.1 \mathrm{mM}$ EDTA, pH 7.0. For NMR studies, the oligomer concentration was brought to $0.6 \mathrm{mM}$, and $\mathrm{H}_{2} \mathrm{O}$ was replaced by either $100 \% \mathrm{D}_{2} \mathrm{O}\left(\mathrm{D}_{2} \mathrm{O}\right.$ sample $)$ or $10 \% \mathrm{D}_{2} \mathrm{O} / 90 \% \mathrm{H}_{2} \mathrm{O}\left(\mathrm{H}_{2} \mathrm{O}\right.$ sample $)$.

${ }^{15} \mathrm{~N},{ }^{13} \mathrm{C}$-labeled RNA of the identical sequence as above was synthesized enzymatically from a synthetic single-stranded DNA template using Escherichia coli $\mathrm{His}_{6}$-tagged RNA polymerase (Milligan et al. 1987; Wyatt et al. 1991) and ${ }^{13} \mathrm{C},{ }^{15} \mathrm{~N}$-labeled rNTPs (Spectra Stable Isotopes). To maximize transcription yield, an $11 \mathrm{nt} 5^{\prime}$-purine-rich sequence tag was included in the DNA template. The resulting $11 \mathrm{nt}$ tag was later removed from the RNA via DNAzyme cleavage (Santoro and Joyce 1997; Cheong et al. 2004). The RNA was purified via $15 \%$ polyacrylamide gel electrophoresis with $6 \mathrm{M}$ urea, identified by brief UV shadowing $(254 \mathrm{~nm})$, and excised from the gel. RNA was recovered by crushing the gel slab followed by a temperature shock (freeze $\left[-80^{\circ} \mathrm{C}\right] /$ thaw $\left.\left[90^{\circ} \mathrm{C}\right]\right)$ treatment, overnight incubation at $37^{\circ} \mathrm{C}$, and either electroelution or diffusion into buffer. Purified RNA samples were concentrated, desalted, and brought to the same buffer conditions as the natural abundance sample via centrifugal filtration.

\section{NMR spectroscopy}

NMR experiments were performed at 600 (Varian Inova), 700, and $800 \mathrm{MHz}$ (Bruker Avance). Each spectrometer is equipped with four rf channels and gradient pulse capabilities. The 700 $\mathrm{MHz}$ spectrometer was equipped with a cryoprobe. In general, spectra of nonexchangeable protons were recorded at $30^{\circ} \mathrm{C}$ with a $99 \% \mathrm{D}_{2} \mathrm{O}$ buffer sample using presaturation to suppress the residual water signal. Initial NOESY spectra were acquired at 600 $\mathrm{MHz}$ with mixing times of $50,75,100,120$, and $400 \mathrm{msec}$ at $25^{\circ} \mathrm{C}$. Typically, a total of $256 t_{1}$ increments were acquired, with 4096 data points and 32 scans per fid and a recycle time of $3 \mathrm{sec}$. To further assist in resonance assignment, 400-msec mixing time NOESY spectra were acquired in $\mathrm{D}_{2} \mathrm{O}$ at a series of temperatures $\left(0,15,25,35^{\circ} \mathrm{C}\right)$, and NOESY spectra in $\mathrm{H}_{2} \mathrm{O}$ were acquired with 50 and 120 msec mixing times at 0 and $5^{\circ} \mathrm{C}$ with the $\mathrm{H}_{2} \mathrm{O}$ signal suppressed by a combination of soft $90^{\circ}$ and z-gradient pulses; $700 \mathrm{MHz}$ NOESY spectra were acquired in $\mathrm{H}_{2} \mathrm{O}$ at $5^{\circ} \mathrm{C}$ with 4096 data points, $200 t_{1}$ increments, 576 scans per fid, and a mixing time of 50 msec with 3-9-19 gradient water suppression, and in
$\mathrm{D}_{2} \mathrm{O}$ with 4096 data points, $464 t_{1}$ increments, and 416 scans per increment with a 300 -msec mixing time.

TOCSY spectra with mixing times of 70 and $200 \mathrm{msec}$ and a DQF-COSY spectrum were recorded at $25^{\circ} \mathrm{C}$ to obtain sugar couplings and through-bond correlations. An ${ }^{1} \mathrm{H},{ }^{13} \mathrm{C}-\mathrm{HMQC}$ spectrum was acquired with spectral widths of 5000 and 10,560 $\mathrm{Hz}$ in the proton and carbon dimensions, respectively. A total of 86 increments were acquired, with 2048 data points, 512 scans per fid, and a recycle time of $3 \mathrm{sec}$. A ${ }^{1} \mathrm{H},{ }^{31} \mathrm{P}$ HETCOR spectrum was acquired with spectral widths of $5000 \mathrm{~Hz}$ in ${ }^{1} \mathrm{H}$ and $800 \mathrm{~Hz}$ in ${ }^{31} \mathrm{P}$. Data were processed with NMRPipe (Delaglio et al. 1995) and visualized and analyzed with NMRview (Johnson and Blevins 1994) or TOPSPIN 1.3 (Bruker). Chemical shift assignments for ${ }^{13} \mathrm{C}$ were based on correlated ${ }^{1} \mathrm{H}$ shifts in the ${ }^{1} \mathrm{H},{ }^{13} \mathrm{C}$-HMQC spectrum and the ${ }^{1} \mathrm{H},{ }^{13} \mathrm{C}$-HSQC experiments described below.

Dipolar couplings were measured at $700 \mathrm{MHz}$ in the ${ }^{13} \mathrm{C}$ dimension of a coupled constant-time HSQC utilizing composite chirp pulses for the inversion of ${ }^{13} \mathrm{C}$ magnetization during the $t_{1}$ period. Where overlap occurred, dipolar couplings were instead obtained from the ${ }^{1} \mathrm{H}$ dimension by removing ${ }^{13} \mathrm{C}$ decoupling during acquisition. Analysis of couplings in both dimensions confirmed that similar measurements were obtained by each method. The measurement precision was estimated by repetition as $\sim 2 \mathrm{~Hz}$. Alignment was achieved by the use of $13 \mathrm{mg} / \mathrm{mL}$ Pf1 phage (Profos), which caused an 11.6-Hz splitting in the deuterium spectrum, indicating a liquid-crystalline state had been obtained.

\section{NMR-derived structural constraints}

NOE-based estimates of the distance between nonexchangeable protons were derived from NOESY cross-peak volumes at mixing times of $50 \mathrm{msec}, 75 \mathrm{msec}$, and $100 \mathrm{msec}$ using an isolated spin pair approximation. Cross-peak volumes were integrated using Felix and build-up rates compared to H5-H6 (2.45 $\AA$ ) cross-peaks and scaled by $1 / r^{6}$, where $r$ represents the distance between the two protons. After preliminary distances were derived in this manner, the potential well minima and maxima were set to this distance $\pm 30 \%$ up to a maximum of $6 \AA$. NOEs involving exchangeable protons were interpreted more conservatively as distance constraints with a similar $+30 \%$ upper limit, but with a lower bound of $1.8 \AA$

Backbone dihedral angles of nucleotides G1-C4, G8-C12, U18C20, and G24-C27 were constrained to A-form helix values $\pm 45^{\circ}$ based on their ${ }^{31} \mathrm{P}$ chemical shifts in the ${ }^{1} \mathrm{H},{ }^{31} \mathrm{P}-\mathrm{HETCOR}$, and their participation in Watson-Crick pairing (Gorenstein 1984). A more cautious interpretation was made for the pyrimidinemismatch region. The alpha and zeta dihedral angles of nucleotides 5-7 and 21-23 were constrained to gauche conformations $\left(0^{\circ} \pm\right.$ $100^{\circ}$ ) based on their ${ }^{31} \mathrm{P}$ shifts (Varani et al. 1991), while other backbone angles in this region were left unconstrained. Backbone constraints were not applied in the triloop region, as the ${ }^{31} \mathrm{P}$ resonances of U13, U15, G16, and U17 are shifted significantly downfield.

A total of 66 well-resolved one-bond RDCs were obtained including $23 \mathrm{~J}_{\mathrm{C}^{\prime}-\mathrm{H}_{1}}, 5 \mathrm{~J}_{\mathrm{C} 2-\mathrm{H} 2}, 15 \mathrm{~J}_{\mathrm{C} 5-\mathrm{H} 5}, 16 \mathrm{~J}_{\mathrm{C} 6-\mathrm{H} 6}, 7 \mathrm{~J}_{\mathrm{C} 8-\mathrm{H} 8} \mathrm{RDCs}$ along with the $\mathrm{J}_{\mathrm{C}^{\prime}-\mathrm{H} 2^{\prime}} \mathrm{RDC}$ of guanidine 16 , which could be resolved on the basis of its unique ${ }^{1} \mathrm{H}$ frequency. The alignment tensors were obtained using an iterative approach similar to that of Warren and Moore (2001). Briefly, a range of values for the rhombicity $(\mathrm{R})$ was generated using the program PALES 
(Zweckstetter and Bax 2000) from a family of structures refined without RDCs. The R values were used to obtain an estimate of the axial component $\left(D_{a}\right)$ of the alignment tensor using the formula

$$
D_{a}=-d_{\max } /(1+1.5 \mathrm{R})
$$

where $d_{\max }$ is the maximum positive value from the histogram of measured RDCs. Although the minima and highest populated values of the histogram can in theory be used to obtain $D_{a}$, in practice these are not adequately sampled in RNA due to the nonrandom distribution of bond angles. However, the value of $d_{\max }$ can be more reliably obtained as it corresponds to $\mathrm{C}-\mathrm{H}$ bonds at an angle of $90^{\circ}$ to the principle alignment tensor, an orientation well sampled by the planar bases of nucleic acids. The tensor values thus obtained were used to generate a set of structures using the RDC constraints. The lowest-energy structures were then used in a second round of alignment tensor prediction with PALES. Structure generation and alignment prediction were further iterated until the tensor values converged. The lowest-energy structures were obtained with $D_{a}=-15.75 \mathrm{~Hz}$ and $\mathrm{R}=0.168$.

\section{Structure calculation}

Structure calculations employed 358 NOE-derived interatomic distance constraints, 85 backbone torsion angle constraints, and 40 hydrogen bond constraints. Initially, hydrogen-bonding constraints were applied only to those base pairs with NOE patterns and observable imino protons indicating participation in standard Watson-Crick pairing (U2-U5, U7-C12, G16-U21, U23-A26). Weak base planarity constraints $\left(10 \mathrm{kcal} \mathrm{mol}^{-1}\right)$ were applied to the Watson-Crick regions and also for U5-U23 and U7-U21 base pairs for which imino-imino NOE peaks were observed, although no assumption was made about the geometry of the pairing. Structure calculations used simulated annealing with Cartesian molecular dynamics in CNS 1.1. HRV-14 SLD structures were generated from a set of helical stem-loops having Watson-Crick base-paired regions but with randomized triloop and pyrimidinemismatch regions. A total of 1200 trial structures were generated, using 4000 steps at $20,000 \mathrm{~K}$ followed by 4000 cooling steps beginning at $1000 \mathrm{~K}$, and then energy minimized using 15 cycles with 200 steps of steepest descent minimization. This produced 510 structures with acceptable geometry, as defined by CNS. The 20 lowest-energy structures unambiguously converged with the $\mathrm{H} 3$ of 5/21 paired with $\mathrm{O} 4$ of $23 / 7$, respectively, and $\mathrm{H} 3$ of $7 / 23$ paired with $\mathrm{O} 2$ of $21 / 5$, respectively. Distance constraints reflecting these hydrogen bonds were therefore applied in subsequent rounds of structure calculation to reinforce appropriate hydrogen bond distances and to increase the efficiency of structure calculation. The first round structures were further refined with or without $66{ }^{1} \mathrm{~J}_{\mathrm{CH}}$ RDC constraints with the same annealing procedure except that the high-temperature stage was reduced to $3000 \mathrm{~K}$. The 10 lowest-energy structures refined with RDCs and having no constraint violations (no NOE or hydrogen bond violation $>0.2 \AA$, no angle violation $>5^{\circ}$, or dipolar coupling violation $>2.0 \mathrm{~Hz}$ ) were selected to represent the solution structure of HRV-14 SLD (Fig. 3A). The 10 lowest-energy structures calculated without RDCs and having no constraint violations (no NOE or hydrogen bond violation $>0.2 \AA$, no angle violation $>5^{\circ}$ ) are shown in Figure $3 \mathrm{~B}$. Structural figures were created with PyMOL (http://pymol.sourceforge.net/).

\section{ACKNOWLEDGMENTS}

The authors thank Thomas Krugh, Douglas Turner, Brent Znosko, Scott Kennedy, Francois Bissey, Gillian Norris, Andrew Sutherland-Smith, and Pat Edwards. Funding for this project was provided in part by the Marsden Fund of the Royal Society of New Zealand.

Received September 25, 2006; accepted October 31, 2006.

\section{References}

Allain, F.H. and Varani, G. 1995. Structure of the P1 helix from group I self-splicing introns. J. Mol. Biol. 250: 333-353.

Amineva, S.P., Aminev, A.G., Palmenberg, A.C., and Gern, J.E. 2004. Rhinovirus $3 \mathrm{C}$ protease precursors $3 \mathrm{CD}$ and $3 \mathrm{CD}^{\prime}$ localize to the nuclei of infected cells. J. Gen. Virol. 85: 2969-2979.

Andino, R., Rieckhof, G.E., Trono, D., and Baltimore, D. 1990. Substitutions in the protease $\left(3 \mathrm{C}^{\mathrm{pro}}\right)$ gene of poliovirus can suppress a mutation in the $5^{\prime}$ noncoding region. J. Virol. 64: 607-612.

Blair, W.S., Parsley, T.B., Bogerd, H.P., Towner, J.S., Semler, B.L., and Cullen, B.R. 1998. Utilization of a mammalian cell-based RNA binding assay to characterize the RNA binding properties of picornavirus 3C proteases. RNA 4: 215-225.

Cheong, H.K., Hwang, E., Lee, C., and Choi, B.S. 2004. Rapid preparation of RNA samples for NMR spectroscopy and X-ray crystallography. Nucleic Acids Res. 32: e84.

Delaglio, F., Greziek, S., Vuister, G.W., Zhu, G., Pfeifer, J., and Bax, A. 1995. NMRPipe: A multidimensional spectral processing system based on UNIX pipes. J. Biomol. NMR 6: 277-293.

Du, Z., Yu, J., Andino, R., and James, T.L. 2003. Extending the family of UNCG-like tetraloop motifs: NMR structure of a CACG tetraloop from coxsackievirus B3. Biochemistry 42: 43734383.

Du, Z., Yu, J., Ulyanov, N.B., Andino, R., and James, T.L. 2004. Solution structure of a consensus stem-loop D RNA domain that plays important roles in regulating translation and replication in enteroviruses and rhinoviruses. Biochemistry 43: 11959-11972.

Gorenstein, D.G., Lai, K., and Shah, D.O. $1984 .{ }^{31} \mathrm{P}$ and twodimensional ${ }^{31} \mathrm{P} /{ }^{1} \mathrm{H}$ correlated NMR spectra of Duplex $\mathrm{d}\left(\mathrm{Ap}\left[{ }^{17} \mathrm{O}\right] \mathrm{Gp}\left[{ }^{18} \mathrm{O}\right] \mathrm{Cp}\left[{ }^{16} \mathrm{O}\right] \mathrm{T}\right)$ and assignment of ${ }^{31} \mathrm{P}$ signals in $\mathrm{d}(\mathrm{ApGpCpT})_{2}$-actinomycin D complex. Biochemistry 23: 67176723.

Greenberg, S.B. 2003. Respiratory consequences of rhinovirus infection. Arch. Intern. Med. 163: 278-284.

Hayden, F.G. 2004. Rhinovirus and the lower respiratory tract. Rev. Med. Virol. 14: 17-31.

Herold, J. and Andino, R. 2001. Poliovirus RNA replication requires genome circularization through a protein-protein bridge. Mol. Cell 7: $581-591$.

Huang, H., Alexandrov, A., Chen, X., Barnes III, T.W., Zhang, H., Dutta, K., and Pascal, S.M. 2001. Structure of an RNA hairpin from HRV-14. Biochemistry 40: 8055-8064.

Johnson, B.A. and Blevins, R.A. 1994. NMRView: A computer program for the visualization and analysis of NMR data. J. Biomol. NMR 4: 603-614.

Johnson, V.A. and Semler, B.L. 1988. Defined recombinants of poliovirus and coxsackievirus: Sequence-specific deletions and functional substitutions in the $5^{\prime}$ noncoding regions of viral RNAs. Virology 162: 47-57.

Kuyumcu-Martinez, N.M., Van Eden, M.E., Younan, P., and Lloyd, R.E. 2004. Cleavage of poly(A)-binding protein by poliovirus $3 \mathrm{C}$ protease inhibits host cell translation: A novel mechanism for host translation shutoff. Mol. Cell. Biol. 24: 1779-1790.

Lawrence, C. and Thatch, R. 1975. Identification of a viral protein involved in post-translational maturation of the encephalomyocarditis virus capsid precursor. J. Virol. 15: 918-928. 
Leong, L.E., Walker, P.A., and Porter, A.G. 1993. Human rhinovirus14 protease $3 \mathrm{C}\left(3 \mathrm{C}^{\mathrm{pro}}\right)$ binds specifically to the $5^{\prime}$-noncoding region of the viral RNA. Evidence that $3 \mathrm{C}^{\text {pro }}$ has different domains for the RNA binding and proteolytic activities. J. Biol. Chem. 268: 25735-25739.

Matthews, D.A., Smith, W.W., Ferre, R.A., Condon, B., Budahazi, G., Sisson, W., Villafranca, J.E., Janson, C.A., McElroy, H.E., and Gribskov, C.L. 1994. Structure of human rhinovirus 3C protease reveals a trypsin-like polypeptide fold, RNA-binding site, and means for cleaving precursor polyprotein. Cell 77: 761-771.

Mauffret, O., Tevanian, G., and Fermandjian, S. 2002. Residual dipolar coupling constants and structure determination of large DNA duplexes. J. Biomol. NMR 24: 317-328.

Meredith, J.M., Rohll, J.B., Almond, J.W., and Evans, D.J. 1999. Similar interactions of the poliovirus and rhinovirus 3D polymerase with the $3^{\prime}$ untranslated region of rhinovirus 14. J. Virol. 73: 9952-9958.

Milligan, J.F., Groebe, D.R., Witherell, G.W., and Uhlenbeck, O.C. 1987. Oligoribonucleotide synthesis using T7 RNA polymerase and synthetic DNA templates. Nucleic Acids Res. 15: 8783-8798.

Ohlenschlager, O., Wohnert, J., Bucci, E., Seitz, S., Hafner, S., Ramachandran, R., Zell, R., and Gorlach, M. 2004. The structure of the stem-loop D subdomain of coxsackievirus B3 cloverleaf RNA and its interaction with the proteinase 3C. Structure 12: 237-248.

Pelham, H.R.B. 1978. Translation of encephalomyocarditis virus RNA in vitro yeilds an active proteolytic processing enzyme. Eur. J. Biochem. 85: 457-462.

Rieder, E., Xiang, W., Paul, A., and Wimmer, E. 2003. Analysis of the cloverleaf element in a human rhinovirus type 14/poliovirus chimera: Correlation of subdomain D structure, ternary protein complex formation and virus replication. J. Gen. Virol. 84: 22032216.
Rohll, J.B., Percy, N., Ley, R., Evans, D.J., Almond, J.W., and Barclay, W.S. 1994. The 5'-untranslated regions of picornavirus RNAs contain independent functional domains essential for RNA replication and translation. J. Virol. 68: 4384-4391.

Santoro, S.W. and Joyce, G.F. 1997. A general purpose RNA-cleaving DNA enzyme. Proc. Natl. Acad. Sci. 94: 4262-4266.

Sarnow, P. 1989. Role of 3 '-end sequence in infectivity of poliovirus transcripts made in vitro. J. Virol. 60: 1040-1049.

Todd, S. and Semler, B.L. 1996. Structure-infectivity analysis of the human rhinovirus genomic RNA 3' non-coding region. Nucleic Acids Res. 24: 2133-2142.

Varani, G., Cheong, C., and Tinoco, I. 1991. Structure of an unusually stable RNA hairpin. Biochemistry 30: 3280-3289.

Walker, P.A., Leong, L.E., and Porter, A.G. 1995. Sequence and structural determinants of the interaction between the $5^{\prime}$-noncoding region of picornavirus RNA and rhinovirus protease 3C. J. Biol. Chem. 270: 14510-14516.

Warren, J.J. and Moore, P.B. 2001. Application of dipolar coupling data to the refinement of the solution structure of the Sarcin-Ricin loop RNA. J. Biomol. NMR 20: 311-323.

Wyatt, J.R., Chastain, M., and Puglisi, J.D. 1991. Synthesis and purification of large amounts of RNA oligonucleotides. Biotechniques 11: 764-769.

Xiang, W., Harris, K.S., Alexander, L., and Wimmer, E. 1995. Interaction between the $5^{\prime}$-terminal cloverleaf and $3 \mathrm{AB} / 3 \mathrm{CD}^{\text {pro }}$ of poliovirus is essential for RNA replication. J. Virol. 69: 3658-3667.

Zell, R., Sidigi, K., Bucci, E., Stelzner, A., and Gorlach, M. 2002. Determinants of the recognition of enteroviral cloverleaf RNA by coxsackievirus B3 proteinase 3C. RNA 8: 188-201.

Zweckstetter, M. and Bax, A. 2000. Prediction of sterically induced alignment in a dilute liquid crystalline phase: Aid to protein structure determination by NMR. J. Am. Chem. Soc. 122: 37913792. 

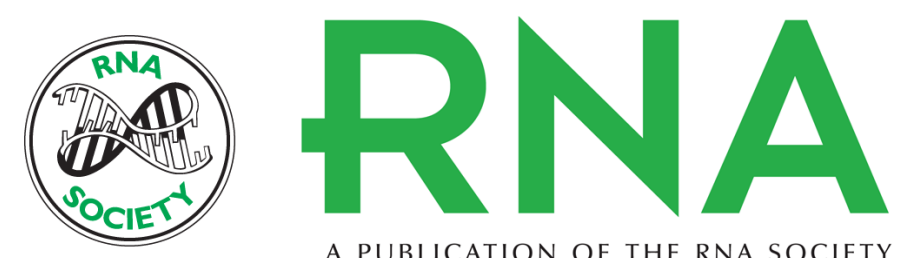

A PUBLICATION OF THE RNA SOCIETY

\section{NMR structure of stem-loop D from human rhinovirus-14}

Stephen J. Headey, He Huang, Jolyon K. Claridge, et al.

RNA 2007 13: 351-360 originally published online December 28, 2006

Access the most recent version at doi:10.1261/rna.313707

References This article cites 36 articles, 11 of which can be accessed free at:

http://rnajournal.cshlp.org/content/13/3/351.full.htmI\#ref-list-1

\section{License}

Email Alerting Receive free email alerts when new articles cite this article - sign up in the box at the Service top right corner of the article or click here. 